\title{
Self-adjointness of the generalized spin-boson Hamiltonian with a quadratic boson interaction
}

\author{
Noriaki TeRANISHI \\ (Received June 11, 2013; Revised November 5, 2013)
}

\begin{abstract}
We consider an abstract model which describes an interaction of nonrelativistic particles with a Bose field. We show that the essential self-adjointness of the generalized spin-boson Hamiltonian with a quadratic boson interaction for all coupling constant and the Hamiltonian is self-adjoint if it is bounded from below under some conditions.
\end{abstract}

Key words: Non-relativistic quamtum field theory, self-adjointness.

\section{Introduction}

In this paper, we consider an abstract model which describes an interaction of non-relativistic particles with a Bose field. Arai and Hirkawa [1] introduced an abstract non-relativistic quantum field model which is a generalization of the spin-boson model and it is called the generalized spin-boson model. Miyao and Sasaki [8] added $\phi^{2}$ term to the generalized spin-boson Hamiltonian. They showed that the Hamiltonian is self-adjoint for small coupling constants by applying the Kato-Rellich theorem. However these restrictions on the coupling constants could be removed because some other non-relativistic quantum field Hamiltonians are self-adjoint for arbitrary coupling constants. For the Nelson models, it is clear since the interaction term is infinitely small with respect to the free Hamiltonian [9]. For the Pauli-Fiertz models, Arai [3] showed that Pauli-Fierz Hamiltonian in the dipole approximation is self-adjoint for arbitrary values of coupling constants by means of the Nelson commutator theorem. Hiroshima [7] proved that the full Pauli-Fierz Hamiltonian is self-adjoint for all coupling constants by using the functional integration. Hasler and Herbst [6] give another proof of the self-adjointness of the Pauli-Fierz Hamiltonian by operator theoretical methods. It is known that the standard spin-boson Hamiltonian is self-adjoint for any coupling constants. 
Arai and Kawano [2] proved the self-adjointness of the Hamiltonian of the generalized spin-boson model for some coupling constants by using an unitary transformation and strong commutativity of some operators. However we hope that the strong commutativity should be removed. Therefore we study the self-adjointness of the Hamiltonian of the generalized spinboson model with a quadratic boson interaction in a more general framework. In particular, we are interested in when the Hamiltonian is (essentially) self-adjoint without assuming the commutativity of some operators.

In this paper, we first prove the essential self-adjointness of a Hamiltonian for all coupling constants under some natural conditions. By using this result, roughly speaking, we also show that semi-boundedness of the Hamiltonian implies the self-adjointness of it. These results improve the existing ones.

The outline of the present paper is as follows. In Section 2, we set up notation and terminology. In the third section, we define the GSB model. In Section 4, we show that the semi-boundedness and some commutativities imply the (essential) self-adjointness of the Hamiltonian. In addition we give a condition for semi-boundedness without strong commutativity. This condition for coupling constants is weaker than the condition which is obtained by using the Kato-Rellich theorem.

\section{Preliminaries}

Let $\mathcal{X}$ be a complex Hilbert space. We denote the inner product and the norm of the Hilbert space $\mathcal{X}$ by $\langle\cdot, \cdot\rangle_{\mathcal{X}}$ and $\|\cdot\|_{\mathcal{X}}$ respectively. For simplicity of notation, we may omit the subscript $\mathcal{X}$ in $\langle\cdot, \cdot\rangle_{\mathcal{X}}$ and $\|\cdot\|_{\mathcal{X}}$ if there is no confusion. In this paper, the inner product is antilinear in the first variable.

For a linear operator $T$ on a Hilbert space $\mathcal{X}$, we denote its domain by $\mathrm{D}(T)$. In this article, "an operator" means "a linear operator". We use standard conventions for the sum and the composition of two operators: $\mathrm{D}(T+S):=\mathrm{D}(T) \cap \mathrm{D}(S)$ and $\mathrm{D}(T S):=\{\Psi \in \mathrm{D}(S) \mid S \Psi \in \mathrm{D}(T)\}$. We recall that an operator $T$ on a Hilbert space is said to be relatively bounded with respect to an operator $S$ or simply $S$-bounded if $\mathrm{D}(S) \subset \mathrm{D}(T)$ and there exist nonnegative constants $a, b \in \mathbb{R}$ such that

$$
\|T \Psi\| \leq a\|S \Psi\|+b\|\Psi\|, \quad \text { for all } \Psi \in \mathrm{D}(S) .
$$


The greatest lower bound $a_{0}$ of all possible constants $a$ in (1) will be called the relative bound of $T$ with respect to $S$.

To describe the Bose fields, one uses the Boson Fock space over a Hilbert space $\mathcal{X}$ :

$$
\begin{aligned}
& \mathscr{F}_{\mathrm{b}}(\mathcal{X}):=\bigoplus_{n=0}^{\infty} \otimes_{\mathrm{s}}^{n} \mathcal{X} \\
& =\left\{\begin{array}{l|l}
\psi=\left\{\psi^{(n)}\right\}_{n=0}^{\infty} & \begin{array}{c}
\text { for all } n \in \mathbb{N}, \psi^{(n)} \in \otimes_{\mathrm{s}}^{n} \mathcal{X} \\
\text { and } \sum_{n=0}^{\infty}\left\|\psi^{(n)}\right\|^{2}<\infty
\end{array}
\end{array}\right\},
\end{aligned}
$$

where $\bigotimes_{\mathrm{s}}^{n} \mathcal{X}$ is the $n$-fold symmetric tensor product of $\mathcal{X}$, i.e., for the symmetrization operator $\mathcal{S}_{n}:=(1 / n !) \sum_{\sigma \in \mathfrak{S}_{n}} U_{\sigma}$ on $\bigotimes^{n} \mathcal{X}$, where $\mathfrak{S}_{n}$ is the symmetric group of degree $n$ and $U_{\sigma}\left(\psi_{1}, \ldots, \psi_{n}\right):=\left(\psi_{\sigma(1)}, \ldots, \psi_{\sigma(n)}\right)$, $\bigotimes_{\mathrm{s}}^{n} \mathcal{X}:=\mathcal{S}_{n}\left(\otimes^{n} \mathcal{X}\right)$ with $\bigotimes_{\mathrm{s}}^{0} \mathcal{X}=\mathbb{C}$. Let us define the finite particle subspace

$$
\begin{aligned}
\mathscr{F}_{\mathrm{b}, 0}(\mathcal{X}): & =\left\{\psi \in \mathscr{F}_{\mathrm{b}}(\mathcal{X}) \mid \begin{array}{c}
\text { there exists a number } n_{0} \in \mathbb{N} \\
\text { such that } \psi^{(n)}=0 \text { for all } n \geq n_{0}
\end{array}\right\} \\
& =\coprod_{n=0}^{\infty} \otimes_{\mathrm{s}}^{n} \mathcal{X} .
\end{aligned}
$$

This is dense in $\mathscr{F}_{\mathrm{b}}(\mathcal{X})$ and a fundamental subspace in the Fock space. Similarly, for a linear subspace $\mathcal{D} \subset \mathcal{X}$, we define a linear subspace $\mathscr{F}_{b}(\mathcal{D}) \subset$ $\mathscr{F}_{b}(\mathcal{X})$;

$$
\mathscr{F}_{\mathrm{b}, \text { fin }}(\mathcal{D}):=\coprod_{n=0}^{\infty} \widehat{\otimes}_{\mathrm{s}}^{n} \mathcal{D} \text {. }
$$

(Here, $\widehat{\otimes}$ is the algebraic tensor product and $\widehat{\otimes}_{\mathrm{s}} \mathcal{X}:=\mathcal{S}_{n}\left(\widehat{\otimes}^{n} \mathcal{X}\right)$.)

Basic objects on $\mathscr{F}_{\mathrm{b}}(\mathcal{X})$ are the creation and annihilation operators. We denote the annihilation operator by $a(f)(f \in \mathcal{X})$ (see, e.g., [10]). The creation operator $a^{*}(f)$ is the adjoint of annihilation operator $a(f)$. For all $f, g \in \mathcal{X}$, these operators obey the canonical commutation relations

$$
\left[a(f), a^{*}(g)\right]=\langle f, g\rangle_{\mathcal{X}}, \quad[a(f), a(g)]=0, \quad\left[a^{*}(f), a^{*}(g)\right]=0
$$


on $\mathscr{F}_{\mathrm{b}, 0}(\mathcal{X})$, where $[X, Y]:=X Y-Y X$.

The Segal field operator is defined as

$$
\phi(f):=\frac{a(f)+a^{*}(f)}{\sqrt{2}}, \quad f \in \mathcal{X} .
$$

This operator $\phi(f)$ is known to be essentially self-adjoint on $\mathscr{F}_{\mathrm{b}, 0}(\mathcal{X})[10$, Theorem X.41 (a)]. We will denote its closure by the same symbol $\phi(f)$. From equalities $(2)$, we have the following identity on $\mathscr{F}_{\mathrm{b}, 0}(\mathcal{X})$,

$$
[\phi(f), \phi(g)]=i \operatorname{Im}\langle f, g\rangle, \quad f, g \in \mathcal{X} .
$$

The second quantization of a densely defined closable operator $S$ is denoted by $\mathrm{d} \Gamma(\mathrm{S})$ and defined by

$$
\mathrm{d} \Gamma(\mathrm{S}):=\bigoplus_{\mathrm{n}=0}^{\infty} \mathrm{S}^{(\mathrm{n})}
$$

where $S^{(n)}$ is defined as follows:

$$
\begin{aligned}
S^{(0)} & :=0 \\
S^{(n)} & :=\overline{\left.\sum_{j=1}^{n} I \otimes \cdots \otimes I \otimes \underset{(j \mathrm{th})}{S} \otimes I \otimes \cdots \otimes I\right|_{\widehat{\otimes}_{s}^{n} D(T)}, \quad \text { if } n \geq 1 .}
\end{aligned}
$$

( $\bar{T}$ means the closure of the operator $T, I$ denotes the identity operator, and $\left.T\right|_{M}$ is the restriction of the operator $T$ on a subspace $M$.) The domain of the second quantization operator $\mathrm{d} \Gamma(S)$ is

$$
\left\{\psi \in \mathcal{F}_{\mathrm{b}}(\mathcal{X}) \mid \psi^{(n)} \in \mathrm{D}\left(S^{(n)}\right), \sum_{n=0}^{\infty}\left\|S^{(n)} \psi^{(n)}\right\|<\infty\right\} .
$$

It is easy to see that, if $S$ is self-adjoint or nonnegative, then so is $\mathrm{d} \Gamma(\mathrm{S})$. The next lemma describes well known properties of $\phi(f)$ and $\mathrm{d} \Gamma(S)$ (see, e.g., [4], [5]).

Lemma 2.1 Let $S$ be a densely defined, injective, nonnegative self-adjoint operator on a Hilbert space $\mathcal{X}$. 
(i ) If $f \in \mathrm{D}\left(S^{-1 / 2}\right)$, then $\mathrm{D}\left(\mathrm{d} \Gamma(S)^{1 / 2}\right) \subseteq \mathrm{D}(\phi(f))$ and

$$
\left\|\phi(f)(\mathrm{d} \Gamma(S)+1)^{-1 / 2}\right\| \leq \sqrt{2}\left(\|f\|+\left\|S^{-1 / 2} f\right\|\right) .
$$

(ii) If $f, g \in \mathrm{D}\left(S^{-1 / 2}\right)$, then $\mathrm{D}(\mathrm{d} \Gamma(S)) \subseteq \mathrm{D}(\phi(f) \phi(g))$ and

$$
\begin{aligned}
& \left\|\phi(f) \phi(g)(\mathrm{d} \Gamma(S)+1)^{-1}\right\| \\
& \quad \leq 4\left(\|f\|+\left\|S^{-1 / 2} f\right\|\right)\left(\|g\|+\left\|S^{-1 / 2} g\right\|\right) .
\end{aligned}
$$

(iii) If $f \in \mathrm{D}(S)$, then

$$
[\mathrm{d} \Gamma(S), \phi(f)]=-i \phi(i S f) \quad \text { on } \mathcal{F}_{\mathrm{b}, 0}(\mathcal{X}) \cap \mathrm{D}(\mathrm{d} \Gamma(S)) .
$$

\section{Definition of a Hamiltonian}

Let $\mathcal{H}$ and $\mathcal{K}$ be Hilbert spaces. We take a Hilbert space

$$
\mathscr{F}:=\mathcal{H} \otimes \mathscr{F}_{\mathrm{b}}(\mathcal{K})
$$

Let $A$ be a self-adjoint operator on $\mathcal{H}$ which is a Hamiltonian of a quantum system, $W$ an injective, self-adjoint and nonnegative operator on $\mathcal{K}$ which is a one-particle Hamiltonian of the Bose field, $B_{j}(j=1, \ldots, n)$ be selfadjoint operators on $\mathcal{H}$ such that $\mathrm{D}(A) \cap \bigcap_{j=1}^{n} D\left(B_{j}\right)$ is dense in $\mathcal{H}, f_{j} \in \mathcal{K}$ $(j=1, \ldots, m), g_{j} \in \mathcal{K}(j=1, \ldots, n)$ and $\lambda, \mu \in \mathbb{R}$. We consider the following operator as the total Hamiltonian of the coupled system:

$$
H(\lambda, \mu):=A \otimes I+I \otimes \mathrm{d} \Gamma(W)+\lambda \sum_{j=1}^{n} B_{j} \otimes \phi\left(g_{j}\right)+\mu \sum_{j=1}^{m} I \otimes \phi\left(f_{j}\right)^{2}
$$

This Hamiltonian $H(\lambda, \mu)$ was studied by Miyao and Sasaki [8]. In the case of $\mu=0$, it was introduced in [1] and called the generalized spin-boson (abbreviated as GSB) Hamiltonian.

In this paper, we need the following conditions.

$\left(\mathrm{H}_{1}\right) \quad A$ is a nonnegative self-adjoint operator on $\mathcal{H}$ and $B_{1}, \ldots, B_{n}$ are $A^{1 / 2}$ bounded symmetric operators.

$\left(\mathrm{H}_{2}\right) W$ is a nonnegative, injective and self-adjoint operator.

$\left(\mathrm{H}_{3}\right)$ There exists a core $\mathrm{D}$ for $A$ such that $\mathrm{D} \subset \bigcap_{j}\left(\mathrm{D}\left(A B_{j}\right) \cap \mathrm{D}\left(B_{j} A\right)\right)$ and 
$\left.\left[A, B_{j}\right]\right|_{\mathrm{D}}$ is $A^{1 / 2}$-bounded for each $j$.

$\left(\mathrm{H}_{4}\right) f_{j}$ and $g_{j} \in \mathrm{D}\left(W^{-1 / 2}\right) \cap \mathrm{D}(W)$ for all $j$

Conditions $\left(\mathrm{H}_{1}\right),\left(\mathrm{H}_{2}\right)$, and $\left(\mathrm{H}_{4}\right)$ are standard condition in the GSB model.

\section{4. (Essential) Self-Adjointness}

In this section, we study the self-adjointness of the operator $H(\lambda, \mu)$. For simplicity, we set

$$
H_{0}:=H(0,0)+1=A \otimes I+I \otimes \mathrm{d} \Gamma(W)+1 .
$$

In what follows, we sometimes write an operator $T$ (resp. $S$ ) for $T \otimes I$ (resp. $I \otimes S)$. Let us first prove that the essential self-ajointness of $H(\lambda, \mu)$.

Proposition 4.1 Suppose that $\left(\mathrm{H}_{1}\right)-\left(\mathrm{H}_{4}\right)$ hold. Then $H(\lambda, \mu)$ is essentially self-adjoint on any core for $H_{0}$.

Proof. Let $D^{\prime}$ be a core for $W$ and $D_{0}:=D \widehat{\otimes} \mathscr{F}_{\mathrm{b}, \text { fin }}\left(D^{\prime}\right)$. Then $D_{0}$ is a core for $H_{0}$. To prove this proposition we use Nelson's commutator theorem [10, Theorem X.37]. We verify that $H(\lambda, \mu)$ and $H_{0}$ satisfy the condition of the commutator theorem. In the following inequalities, $C$ denotes a constant which may change from one inequality to the next. By using (i) and (ii) in Lemma 2.1, we have the following inequalities for all $\Psi \in D_{0}$,

$$
\begin{aligned}
\|H(\lambda, \mu) \Psi\| \leq & \|(A \otimes I+I \otimes \mathrm{d} \Gamma(W)) \Psi\|+|\lambda| \sum_{j=1}^{n}\left\|B_{j} \otimes \phi\left(g_{j}\right) \Psi\right\| \\
& +|\mu| \sum_{j=1}^{m}\left\|I \otimes \phi\left(f_{j}\right)^{2} \Psi\right\| \\
\leq & \|(A \otimes I+I \otimes \mathrm{d} \Gamma(W)+1) \Psi\| \\
& +C \sum_{j=1}^{n}\left\|(A+1)^{1 / 2} \otimes(\mathrm{d} \Gamma(W)+1)^{1 / 2} \Psi\right\| \\
& +C \sum_{j=1}^{m}\|I \otimes(\mathrm{d} \Gamma(W)+1) \Psi\| \\
\leq & \left\|H_{0} \Psi\right\|+C\langle(A+1) \otimes I \Psi, I \otimes(\mathrm{d} \Gamma(W)+1) \Psi\rangle^{1 / 2}+C\left\|H_{0} \Psi\right\|
\end{aligned}
$$




$$
\begin{aligned}
& \leq C\left\|H_{0} \Psi\right\|+C\|(A+1) \otimes I \Psi\|^{1 / 2}\|I \otimes(\mathrm{d} \Gamma(W)+1) \Psi\|^{1 / 2} \\
& \leq C\left\|H_{0} \Psi\right\|+C(\|(A+1) \otimes I \Psi\|+\|I \otimes(\mathrm{d} \Gamma(W)+1) \Psi\|) \\
& \leq C\left\|H_{0} \Psi\right\|
\end{aligned}
$$

Similarly,

$$
\begin{aligned}
& \left|\left\langle H(\lambda, \mu) \Psi, H_{0} \Psi\right\rangle-\left\langle H_{0} \Psi, H(\lambda, \mu) \Psi\right\rangle\right| \\
& \leq|\lambda| \sum_{j=1}^{n}\left|\left\langle B_{j} \otimes \phi\left(g_{j}\right) \Psi, H_{0} \Psi\right\rangle-\left\langle H_{0} \Psi, B_{j} \otimes \phi\left(g_{j}\right) \Psi\right\rangle\right| \\
& +|\mu| \sum_{j=1}^{m}\left|\left\langle\phi\left(f_{j}\right)^{2} \Psi, \mathrm{d} \Gamma(W) \Psi\right\rangle-\left\langle\mathrm{d} \Gamma(W) \Psi, \phi\left(f_{j}\right)^{2} \Psi\right\rangle\right| \\
& \leq|\lambda| \sum_{j=1}^{n}\left|\left\langle I \otimes \phi\left(g_{j}\right) \Psi,\left[B_{j}, A\right] \Psi\right\rangle+\left\langle B_{j} \Psi, I \otimes\left[\phi\left(g_{j}\right), \mathrm{d} \Gamma(W)\right] \Psi\right\rangle\right| \\
& +|\mu| \sum_{j=1}^{m}\left|\left\langle\left[\phi\left(f_{j}\right), \mathrm{d} \Gamma(W)\right] \Psi, \phi\left(f_{j}\right) \Psi\right\rangle-\left\langle\phi\left(f_{j}\right) \Psi,\left[\mathrm{d} \Gamma(W), \phi\left(f_{j}\right)\right] \Psi\right\rangle\right| \\
& \leq|\lambda| \sum_{j=1}^{n}\left(\left\|I \otimes \phi\left(g_{j}\right) \Psi\right\|\left\|\left[B_{j}, A\right] \Psi\right\|+\left\|B_{j} \Psi\right\|\left\|I \otimes \phi\left(i W g_{j}\right) \Psi\right\|\right) \\
& +|\mu| \sum_{j=1}^{m}\left|\left\langle\phi\left(i W f_{j}\right) \Psi, \phi\left(f_{j}\right) \Psi\right\rangle+\left\langle\phi\left(f_{j}\right) \Psi, \phi\left(i W f_{j}\right) \Psi\right\rangle\right| \\
& \leq|\lambda| C \sum_{j=1}^{n}\left\|I \otimes(\mathrm{d} \Gamma(W)+1)^{1 / 2} \Psi\right\|\left(\left\|\left[B_{j}, A\right] \Psi\right\|+\left\|B_{j} \Psi\right\|\right) \\
& +|\mu| C \sum_{j=1}^{m}\left\|I \otimes(\mathrm{d} \Gamma(W)+1)^{1 / 2} \Psi\right\|^{2} \\
& \leq 2 n|\lambda| C\left\|I \otimes(\mathrm{d} \Gamma(W)+1)^{1 / 2} \Psi\right\|\left\|(A+1)^{1 / 2} \otimes I \Psi\right\| \\
& +m|\mu| C\left\|I \otimes(\mathrm{d} \Gamma(W)+1)^{1 / 2} \Psi\right\|^{2} \\
& \leq(2 n|\lambda|+m|\mu|) C\left\|H_{0}^{1 / 2} \Psi\right\|^{2} \text {. }
\end{aligned}
$$


By Nelson's commutator theorem, the operator $H(\lambda, \mu)$ is essentially selfadjoint on the subspace $D$ and any core for $H_{0}$.

We next show the self-adjointness of $H(\lambda, \mu)$. From the previous proposition, we see that $H(\lambda, \mu)$ is essentially self-adjoint on the domain $\mathrm{D}\left(H_{0}\right)$ for any coupling constant under some condition. We infer that $H(\lambda, \mu)$ is self-adjoint for any coupling constant under suitable condition even if $A$ and $B_{j}$ are not commutative. Here we do not show that the self-adjontness of the Hamiltonian for all coupling constant. However, in the next theorem, we prove that the self-adjointness of $H(\lambda, \mu)$ follows from the semi-boundedness of $H(\lambda, 0)$ under natural conditions.

Theorem 4.2 Suppose that $\left(\mathrm{H}_{1}\right)-\left(\mathrm{H}_{3}\right)$ hold. Assume, in addition, the following conditions hold:

( i ) the core $D \subset \bigcap_{j} \mathrm{D}\left(A B_{j}\right) \cap \mathrm{D}\left(A^{2}\right)$ and $\left.\left[A^{1 / 2}, B_{j}\right]\right|_{\mathrm{D}}$ is bounded;

(ii) $f_{j}, g_{j} \in \mathrm{D}\left(W^{-1 / 2}\right) \cap \mathrm{D}\left(W^{2}\right)$ for all $j$.

If $H(\lambda, 0)$ is bounded from below for some $\lambda$, then for all $\lambda^{\prime}$ with $\left|\lambda^{\prime}\right|<|\lambda|$ and $\mu \geq 0, H\left(\lambda^{\prime}, \mu\right)$ is a self-adjoint operator and $\mathrm{D}\left(H\left(\lambda^{\prime}, \mu\right)\right)=\mathrm{D}\left(H_{0}\right)$.

Proof. Without loss of generality, we can assume that $0<\lambda^{\prime}<\lambda$. It follows from Proposition 4.1 that $H\left(\lambda^{\prime}, \mu\right)$ is essentially self-adjoint on $\mathrm{D}\left(H_{0}\right)$ and $\mathrm{D}\left(H_{0}\right) \subseteq \mathrm{D}\left(\overline{H\left(\lambda^{\prime}, \mu\right)}\right)$.

Hence we only have to verify that $\mathrm{D}\left(\overline{H\left(\lambda^{\prime}, \mu\right)}\right) \subseteq \mathrm{D}\left(H_{0}\right)$. Since $0<\lambda^{\prime}<$ $\lambda$, there exists a positive number $\eta<1$ such that $\lambda=\lambda^{\prime} /(1-\eta)$. Let $D^{\prime}$ be a core for $W^{2}, D_{0}:=D \widehat{\otimes} \mathscr{F}_{\mathrm{b}, \text { fin }}\left(D^{\prime}\right), \mu^{\prime}:=\mu /(1-\eta), c$ the infimum of the spectrum of $H(\lambda, 0)$, that is, $c:=\inf \sigma(H(\lambda, 0))$, and $\gamma:=\sup \left\|\overline{\left[A^{1 / 2}, B_{j}\right]}\right\|$. For all $\Psi \in D_{0}$,

$$
\begin{aligned}
\|( & \left.H\left(\lambda^{\prime}, \mu\right)+\eta\right) \Psi\left\|^{2}-\frac{\eta^{2}}{2}\right\| H_{0} \Psi \|^{2} \\
\geq & \frac{\eta^{2}}{2}\left\|H_{0} \Psi\right\|^{2}+(1-\eta)^{2}\left\|H\left(\lambda, \mu^{\prime}\right) \Psi\right\|^{2} \\
& +\eta(1-\eta)\left(\left\langle H_{0} \Psi, H\left(\lambda, \mu^{\prime}\right) \Psi\right\rangle+\left\langle H\left(\lambda, \mu^{\prime}\right) \Psi, H_{0} \Psi\right\rangle\right) \\
\geq & \frac{\eta^{2}}{2}\left\|H_{0} \Psi\right\|^{2}
\end{aligned}
$$




$$
\begin{aligned}
& +\eta(1-\eta)\left(2\left\langle A^{1 / 2} \Psi, H(\lambda, 0) A^{1 / 2} \Psi\right\rangle+2 \operatorname{Re}\left\langle A^{1 / 2} \Psi,\left[A^{1 / 2}, H(\lambda, 0)\right] \Psi\right\rangle\right. \\
& +2\left\langle(\mathrm{~d} \Gamma(W)+1)^{1 / 2} \Psi, H(\lambda, 0)(\mathrm{d} \Gamma(W)+1)^{1 / 2} \Psi\right\rangle \\
& \left.+2 \operatorname{Re}\left\langle(\mathrm{d} \Gamma(W)+1)^{1 / 2} \Psi,\left[(\mathrm{d} \Gamma(W)+1)^{1 / 2}, H(\lambda, 0)\right] \Psi\right\rangle\right) \\
& +\eta(1-\eta) \mu^{\prime} \sum_{j=1}^{m} 2 \operatorname{Re}\left\langle(\mathrm{d} \Gamma(W)+1) \Psi, \phi\left(f_{j}\right)^{2} \Psi\right\rangle
\end{aligned}
$$

By the semi-boundedness of $H(\lambda, 0)$, we have

$$
\begin{gathered}
\left\langle A^{1 / 2} \Psi, H(\lambda, 0) A^{1 / 2} \Psi\right\rangle \geq c\left\|A^{1 / 2} \Psi\right\|^{2}, \\
\left\langle(\mathrm{~d} \Gamma(W)+1)^{1 / 2} \Psi, H(\lambda, 0)(\mathrm{d} \Gamma(W)+1)^{1 / 2} \Psi\right\rangle \geq c\left\|(\mathrm{~d} \Gamma(W)+1)^{1 / 2} \Psi\right\|^{2} .
\end{gathered}
$$

Hence we get the following inequality.

$$
\begin{aligned}
&\left\|\left(H\left(\lambda^{\prime}, \mu\right)+\eta\right) \Psi\right\|^{2}-\frac{\eta^{2}}{2}\left\|H_{0} \Psi\right\|^{2} \\
& \geq \frac{\eta^{2}}{2}\left\|H_{0} \Psi\right\|^{2} \\
&+\eta(1-\eta)\left(2 c\left\|A^{1 / 2} \Psi\right\|^{2}+2 c\left\|(\mathrm{~d} \Gamma(W)+1)^{1 / 2} \Psi\right\|^{2}\right) \\
&-2 \eta(1-\eta)\left\|A^{1 / 2} \Psi\right\|\left\|\left[H(\lambda, 0), A^{1 / 2}\right] \Psi\right\| \\
&+\eta(1-\eta)\left\langle\Psi,\left[(\mathrm{d} \Gamma(W)+1)^{1 / 2},\left[(\mathrm{~d} \Gamma(W)+1)^{1 / 2}, H(\lambda, 0)\right]\right] \Psi\right\rangle \\
&+2 \eta(1-\eta) \mu^{\prime} \sum_{j=1}^{m}\left\langle\phi\left(f_{j}\right) \Psi,(\mathrm{d} \Gamma(W)+1) \phi\left(f_{j}\right) \Psi\right\rangle \\
&+2 \eta(1-\eta) \mu^{\prime} \sum_{j=1}^{m} \operatorname{Re}\left\langle\left[\phi\left(f_{j}\right), \mathrm{d} \Gamma(W)\right] \Psi, \phi\left(f_{j}\right) \Psi\right\rangle \\
& \geq \frac{\eta^{2}}{2}\left\|H_{0} \Psi\right\|^{2} \\
&+2 c \eta(1-\eta)\left(\left\|A^{1 / 2} \Psi\right\|^{2}+\left\|(\mathrm{d} \Gamma(W)+1)^{1 / 2} \Psi\right\|^{2}\right) \\
&-2 \eta(1-\eta)|\lambda| \sum_{j=1}^{n}\left\|A^{1 / 2} \Psi\right\|\left\|\left[A^{1 / 2}, B_{j}\right] \otimes \phi\left(g_{j}\right) \Psi\right\|
\end{aligned}
$$




$$
\begin{aligned}
& -\eta(1-\eta)|\lambda| \sum_{j=1}^{n}\left\|B_{j} \Psi\right\| \\
& \times\left\|\left[(\mathrm{d} \Gamma(W)+1)^{1 / 2},\left[(\mathrm{~d} \Gamma(W)+1)^{1 / 2}, \phi\left(g_{j}\right)\right]\right] \Psi\right\| \\
& -\eta(1-\eta)\left|\mu^{\prime}\right| \sum_{j=1}^{m}\|\Psi\|\left\|\left[\left[\mathrm{d} \Gamma(W), \phi\left(f_{j}\right)\right], \phi\left(f_{j}\right)\right] \Psi\right\| .
\end{aligned}
$$

In the following arguments, we show that each term of (8)-(10) is greater than or equal to $-C\left(\left\|A^{1 / 2} \Psi\right\|^{2}+\left\|I \otimes(\mathrm{d} \Gamma(W)+1)^{1 / 2} \Psi\right\|^{2}\right)$ for some positive constant $C$.

First, we estimate the term (8). Since $\left[A^{1 / 2}, B_{j}\right]$ is bounded,

$$
\begin{aligned}
& \left\|A^{1 / 2} \Psi\right\|\left\|\left[A^{1 / 2}, B_{j}\right] \otimes \phi\left(g_{j}\right) \Psi\right\| \\
& \quad \leq \gamma\left\|A^{1 / 2} \Psi\right\|\left\|I \otimes \phi\left(g_{j}\right) \Psi\right\| \\
& \quad \leq C\left\|A^{1 / 2} \Psi\right\|\left\|(\mathrm{d} \Gamma(W)+1)^{1 / 2} \Psi\right\| \\
& \quad \leq C\left(\left\|A^{1 / 2} \Psi\right\|^{2}+\left\|(\mathrm{d} \Gamma(W)+1)^{1 / 2} \Psi\right\|^{2}\right) .
\end{aligned}
$$

Next, we consider the term (9). It is known that for a nonnegative self-adjoint operator $T$ on a Hilbert space,

$$
T^{1 / 2} \psi=\left(\frac{1}{\pi} \int_{0}^{\infty} \lambda^{-1 / 2}(T+\lambda)^{-1} d \lambda\right) T \psi
$$

for any $\psi \in \mathrm{D}(T)$ (see [11, Chapter VIII Problem 50 (c)]). Since $\Psi \in D_{0}$ and $g_{j} \in \mathrm{D}\left(W^{2}\right)$, it is easy to see that $(\mathrm{d} \Gamma(W)+1)^{1 / 2} \Psi, \phi\left(g_{j}\right) \Psi, \phi\left(g_{j}\right)(\mathrm{d} \Gamma(W)+$ $1)^{1 / 2} \Psi$, and $\left[(\mathrm{d} \Gamma(W)+1)^{1 / 2}, \phi\left(g_{j}\right)\right] \Psi$ are in $\mathrm{D}(\mathrm{d} \Gamma(W))$. Using the formula (11), we can calculate as follow.

$$
\begin{aligned}
& {\left[(\mathrm{d} \Gamma(W)+1)^{1 / 2}, \quad\left[(\mathrm{~d} \Gamma(W)+1)^{1 / 2}, \phi\left(g_{j}\right)\right]\right] \Psi} \\
& =\frac{1}{\pi^{2}} \int_{0}^{\infty} d t \int_{0}^{\infty} d s \frac{1}{\sqrt{t s}}\left[(\mathrm{~d} \Gamma(W)+1+s)^{-1}(\mathrm{~d} \Gamma(W)+1),\right. \\
& \left.\quad\left[(\mathrm{d} \Gamma(W)+1+t)^{-1}(\mathrm{~d} \Gamma(W)+1), \phi\left(g_{j}\right)\right]\right] \Psi
\end{aligned}
$$




$$
\begin{aligned}
= & \frac{1}{\pi^{2}} \int_{0}^{\infty} d t \int_{0}^{\infty} d s \sqrt{t s}(\mathrm{~d} \Gamma(W)+1+s)^{-1}(\mathrm{~d} \Gamma(W)+1+t)^{-1} \\
& \times \phi\left(W^{2} g_{j}\right)(\mathrm{d} \Gamma(W)+1+t)^{-1}(\mathrm{~d} \Gamma(W)+1+s)^{-1} \Psi .
\end{aligned}
$$

Thus we get a bound of the term (9) from the following computation:

$$
\begin{aligned}
&\left\|\left[(\mathrm{d} \Gamma(W)+1)^{1 / 2},\left[(\mathrm{~d} \Gamma(W)+1)^{1 / 2}, \phi\left(g_{j}\right)\right]\right] \Psi\right\| \\
& \leq \frac{1}{\pi^{2}} \int_{0}^{\infty} d t \int_{0}^{\infty} d s \frac{\sqrt{t s}}{(1+t)(1+s)} \\
& \times\left\|\phi\left(W^{2} g_{j}\right)(\mathrm{d} \Gamma(W)+1+t)^{-1}(\mathrm{~d} \Gamma(W)+1+s)^{-1} \Psi\right\| \\
& \leq C\left(\frac{1}{\pi} \int_{0}^{\infty} d s \frac{\sqrt{s}}{(1+s)^{2}}\right)^{2}\left\|(\mathrm{~d} \Gamma(W)+1)^{1 / 2} \Psi\right\| .
\end{aligned}
$$

Using Lemma 2.1 (iii) and the identity (3), we have an inequality about the term (10),

$$
\left|\left\langle\Psi,\left[\left[\mathrm{d} \Gamma(W), \phi\left(f_{j}\right)\right], \phi\left(f_{j}\right)\right] \Psi\right\rangle\right| \leq\left|\left\langle W f_{j}, f_{j}\right\rangle\right|\|\Psi\|^{2} .
$$

Hence we see that

$$
\begin{aligned}
& \left\|\left(H\left(\lambda^{\prime}, \mu\right)+\eta\right) \Psi\right\|^{2}-\frac{\eta^{2}}{2}\left\|H_{0} \Psi\right\|^{2} \\
& \quad \geq \frac{\eta^{2}}{2}\left\|H_{0} \Psi\right\|^{2}-C\left(\left\|A^{1 / 2} \Psi\right\|^{2}+\left\|(\mathrm{d} \Gamma(W)+1)^{1 / 2} \Psi\right\|^{2}+\|\Psi\|^{2}\right) \\
& \quad=\frac{\eta^{2}}{2}\left\|H_{0} \Psi\right\|^{2}-C\left\langle\Psi, H_{0} \Psi\right\rangle-C\|\Psi\|^{2} \\
& \quad \geq-C\|\Psi\|^{2} .
\end{aligned}
$$

Since $D_{0}$ is a core for $H\left(\lambda^{\prime}, \mu\right)$, the above inequality implies

$$
\mathrm{D}\left(\overline{H\left(\lambda^{\prime}, \mu\right)}\right) \subseteq \mathrm{D}\left(H_{0}\right) .
$$

Thus $H\left(\lambda^{\prime}, \mu\right)$ is self-adjoint and $\mathrm{D}\left(H\left(\lambda^{\prime}, \mu\right)\right)=\mathrm{D}\left(H_{0}\right)$.

Our next aim is to prove the semi-boundedness of $H(\lambda, 0)$. Next proposition gives us a sufficient condition. To simplify notation, we write 


$$
H(\lambda):=H(\lambda, 0)=A+\mathrm{d} \Gamma(W)+\lambda \sum_{j=1}^{n} B_{j} \otimes \phi\left(g_{j}\right)
$$

Proposition 4.3 Let $A$ be a nonnegative self-adjoint operator on the Hilbert space $\mathcal{H},\left\{B_{j}\right\}_{j=1}^{n}$ is a family of self-adjoint operators on $\mathcal{H}$, and $W$ a nonnegative injective self-adjoint operator on the Hilbert space $\mathcal{K}$. Suppose that $A$ has a decomposition $A=\sum_{j=1}^{n} A_{j}$ satisfying following conditions:

(i ) the operator $A_{j}$ is a nonnegative self-adjoint operator with $\mathrm{D}(A) \subset$ $\mathrm{D}\left(A_{j}\right)$ for all $j$;

(ii) each operator $B_{j}$ is $A_{j}^{1 / 2}$-bounded;

(iii) there exists a dense subset $D_{j} \subset \mathrm{D}\left(A_{j} B_{j}\right) \cap \mathrm{D}\left(B_{j} A_{j}\right)$ which is a core for $A_{j}$ and the commutator $\left[A_{j}, B_{j}\right]$ on $D_{j}$ is bounded for each $j$.

If $g_{j} \in \mathrm{D}\left(W^{-3 / 2}\right) \cap \mathrm{D}(W), e^{i t B_{j} \otimes \phi\left(i W^{-1} g_{j}\right)}\left(\mathrm{D}\left(A_{j} \otimes I\right)\right) \subseteq \mathrm{D}\left(A_{j} \otimes I\right)$ for all $t \in \mathbb{R}$ and $j$, and there exists a $\left\{\lambda_{j}\right\}_{j=1}^{n}$ such that $0<\lambda_{j}<1, \sum_{j=1}^{n} \lambda_{j}=1$, and $A_{j}-\lambda^{2} \lambda_{j}^{-1}\left\|W^{-1 / 2} g_{j}\right\|^{2} B_{j}^{2} / 2$ is bounded from below for each $j$, then $H(\lambda)$ is bounded from below.

Proof. We first prove that $\mathrm{D}\left(A_{j}\right) \subseteq \mathrm{D}\left(B_{j}^{2}\right)$ for all $j$. Since $B_{j}$ is an $A_{j}^{1 / 2}$ bounded operator, there exist positive constants $c_{j}$ and $d_{j}$ such that

$$
\left\|B_{j} \Psi\right\| \leq c_{j}\left\|A_{j}^{1 / 2} \Psi\right\|+d_{j}\|\Psi\| \quad \text { for all } \Psi \in \mathrm{D}\left(A_{j}^{1 / 2}\right) .
$$

Hence, for all $\Psi \in D_{j} \subset \mathrm{D}\left(A_{j} B_{j}\right) \cap \mathrm{D}\left(B_{j} A_{j}\right)$ and $\varepsilon>0$,

$$
\begin{aligned}
\left\|B_{j}^{2} \Psi\right\| \leq & c_{j}\left\|A_{j}^{1 / 2} B_{j} \Psi\right\|+d_{j}\left\|B_{j} \Psi\right\| \\
\leq & c_{j}\left(\left\|\left[\left(A_{j}+1\right)^{1 / 2}, B_{j}\right] \Psi\right\|+\left\|B_{j}\left(A_{j}+1\right)^{1 / 2} \Psi\right\|\right) \\
& +d_{j}\left(c_{j}\left\|A_{j}^{1 / 2} \Psi\right\|+d_{j}\|\Psi\|\right) \\
\leq & \left(c_{j}^{2}+2 \varepsilon c_{j} d_{j}\right)\left\|A_{j} \Psi\right\| \\
& +\left(d_{j}^{2}+2 c_{j} d_{j} c(\varepsilon)+c_{j}^{2}+c_{j} \frac{1}{\pi} \int_{0}^{\infty} \frac{\sqrt{t}}{(1+t)^{2}} d t\left\|\left[A_{j}, B_{j}\right]\right\|\right)\|\Psi\| .
\end{aligned}
$$

Here $c(\varepsilon)$ is a constant depending on $\varepsilon$ which satisfies 


$$
\left\|\left(A_{j}+1\right)^{1 / 2} \Psi\right\| \leq \varepsilon\left\|A_{j} \Psi\right\|+c(\varepsilon)\|\Psi\|, \quad \text { for all } \Psi \in \mathrm{D}\left(A_{j}\right) .
$$

Since $D_{j}$ is a core for $A_{j}, B_{j}^{2}$ is an $A_{j}$-bounded operator. From this it follows that $\mathrm{D}\left(A_{j}\right) \subseteq \mathrm{D}\left(B_{j}^{2}\right)$ for all $j$.

We set

$$
\begin{aligned}
H_{0 j}(\lambda) & :=A_{j}+\lambda_{j} \mathrm{~d} \Gamma(W)+\lambda B_{j} \otimes \phi\left(g_{j}\right), \\
U_{j}(\lambda) & :=\exp \left(i \lambda \lambda_{j}^{-1} B_{j} \otimes \phi\left(i W^{-1} g_{j}\right)\right) .
\end{aligned}
$$

From an argument similar to that in Lemma 3.7 in [2], it follows that

$$
U_{j}(-\lambda) H_{0 j}(\lambda) U_{j}(\lambda)=A_{j}-\frac{\lambda^{2}}{2 \lambda_{j}}\left\|W^{-1 / 2} g_{j}\right\|^{2} B_{j}^{2}+\lambda_{j} \mathrm{~d} \Gamma(W)+\delta A_{j}(\lambda) .
$$

Here $\delta A_{j}(\lambda):=U_{j}(-\lambda)\left(A_{j} \otimes I\right) U_{j}(\lambda)-A_{j} \otimes I$. We see that $\delta A_{j}(\lambda)$ is infinitesimally small with respect to $I \otimes \mathrm{d} \Gamma(W)$. Indeed, by assumption of $g_{j} \in \mathrm{D}\left(W^{-3 / 2}\right)$ and boundedness of $\left[A_{j}, B_{j}\right]$, the same proof of Lemma 3.10 in [2] works for $\delta A_{j}(\lambda)$. Since unitary transformation preserve the spectral property, by the Kato-Rellich theorem, we see that $H_{0 j}(\lambda)$ is bounded from below for each $j$. Hence $H(\lambda)=\sum H_{0 j}(\lambda)$ is bounded from below.

Corollary 4.4 Suppose that $\left(\mathrm{H}_{1}\right)-\left(\mathrm{H}_{3}\right)$ hold. Assume, in addition, the following conditions hold:

(i ) $B_{j}$ is self-adjoint and $e^{i t B_{j} \otimes \phi\left(i W^{-1} g_{j}\right)}(\mathrm{D}(A \otimes I)) \subseteq \mathrm{D}(A \otimes I)$ for all $t \in \mathbb{R}$ and $j$;

(ii) $\left.\left[A, B_{j}\right]\right|_{D}$ is a bounded operator for each $j$;

(iii) $g_{j} \in \mathrm{D}\left(W^{-3 / 2}\right) \cap \mathrm{D}(W)$ for all $j$.

If there exists $a\left\{\lambda_{j}\right\}_{j=1}^{n}$ such that $0<\lambda_{j}<1, \sum_{j=1}^{n} \lambda_{j}=1$, and $A-\lambda^{2} \lambda_{j}^{-2}\left\|W^{-1 / 2} g_{j}\right\|^{2} B_{j}^{2} / 2$ is bounded from below for each $j$, then $H(\lambda)$ is bounded from below.

Proof. Applying Proposition 4.3 with $A_{j}=\lambda_{j} A$ for each $j$.

Remark 4.5 Under the conditions of Corollary 4.4 except for existence of the $\left\{\lambda_{j}\right\}_{j=1}^{n}$, if $|\lambda| \sum_{j=1}^{n} c_{j}\left\|W^{-1 / 2} g_{j}\right\|<\sqrt{2}$, then there exists a $\left\{\lambda_{j}\right\}_{j=1}^{n}$ such that $0<\lambda_{j}<1, \sum_{j=1}^{n} \lambda_{j}=1$, and $A-\lambda^{2} \lambda_{j}^{-2}\left\|W^{-1 / 2} g_{j}\right\|^{2} B_{j}^{2} / 2$ is self-adjoint and bounded from below for all $j$. Here $c_{j}$ is an $A^{1 / 2}$-bound of 
$B_{j}$. Indeed, there exists a $\left\{\lambda_{j}\right\}_{j=1}^{n}$ such that $0<\lambda_{j}<1, \sum_{j=1}^{n} \lambda_{j}=1$, and $|\lambda| c_{j}\left\|W^{-1 / 2} g_{j}\right\|<\sqrt{2} \lambda_{j}$ for all $j$ whenever $\lambda$ satisfies

$$
|\lambda| \sum_{j=1}^{n} c_{j}\left\|W^{-1 / 2} g_{j}\right\|<\sqrt{2} .
$$

From the proof of Proposition 4.3, we see that relative bound of $B_{j}^{2}$ with respect to $A$ is less than or equal to $c_{j}^{2}$. Therefore, from the Kato-Rellich theorem, we have the desired $\left\{\lambda_{j}\right\}_{j=1}^{n}$. This condition is weaker than the condition (A.3) in [1].

Finally, from the above results, we obtain the following corollary.

Corollary 4.6 Suppose that $\left(\mathrm{H}_{1}\right),\left(\mathrm{H}_{2}\right)$ and $\left(\mathrm{H}_{4}\right)$ hold. Assume, in addition, the following conditions hold:

(i ) $B_{j}$ is self-adjoint and $e^{i t B_{j} \otimes \phi\left(i W^{-1} g_{j}\right)}(\mathrm{D}(A \otimes I)) \subseteq \mathrm{D}(A \otimes I)$ for all $t \in \mathbb{R}$ and $j$

(ii) there exists a core $D$ for $A$ such that $D \subset \mathrm{D}\left(A^{2}\right) \cap \bigcap_{j} \mathrm{D}\left(A B_{j}\right)$ and $\left.\left[A, B_{j}\right]\right|_{D}$ is a bounded operator for each $j$;

If $g_{j} \in \mathrm{D}\left(W^{-3 / 2}\right) \cap \mathrm{D}\left(W^{2}\right)$ for all $j,|\lambda| \sum_{j=1}^{n} c_{j}\left\|W^{-1 / 2} g_{j}\right\|<\sqrt{2}$, and $\mu \geq 0$, then $H(\lambda, \mu)$ is a self-adjoint operator with $\mathrm{D}(H(\lambda, \mu))=\mathrm{D}(H(0,0))$.

Proof. In the proof of Theorem 4.2, we can replace the term $A^{1 / 2}$ by $(A+1 / 2)^{1 / 2}$ and $(\mathrm{d} \Gamma(W)+1)^{1 / 2}$ by $(\mathrm{d} \Gamma(W)+1 / 2)^{1 / 2}$. Using the formlua (11), it is easy to see that $\left.\left[(A+1 / 2)^{1 / 2}, B_{j}\right]\right|_{D}$ is bounded. Therefore we see that similar argument in the proof of Theorem 4.2 work. From this and Corollary 4.4 and Remark 4.5, we have the self-adjointness of $H(\lambda, \mu)$ and $\mathrm{D}(H(\lambda, \mu))=\mathrm{D}(H(0,0))$.

\section{References}

[ 1 ] Arai A. and Hirokawa M., On the existence and uniqueness of ground states of a generalized spin-boson model. J. Funct. Anal., 151(2) (1997), 455-503.

[2] Arai A. and Kawano H., Enhanced binding in a general class of quantum field models. Rev. Math. Phys., 15(4) (2003), 387-423.

[ 3 ] Arai A., A note on scattering theory in nonrelativistic quantum electrodynamics. J. Phys. A, 16(1) (1983), 49-69. 
[ 4 ] Arai A., Perturbation of embedded eigenvalues: a general class of exactly soluble models in Fock spaces. Hokkaido Math. J., 19(1) (1990), 1-34.

[ 5 ] Hasler D. and Herbst I., Absence of ground states for a class of translation invariant models of non-relativistic QED. Comm. Math. Phys., 279(3) (2008), 769-787.

[ 6 ] Hasler D. and Herbst I., On the self-adjointness and domain of Pauli-Fierz type Hamiltonians. Rev. Math. Phys., 20(7) (2008), 787-800.

[ 7 ] Hiroshima F., Self-adjointness of the Pauli-Fierz Hamiltonian for arbitrary values of coupling constants. Ann. Henri Poincaré, 3(1) (2002), 171-201.

[ 8 ] Miyao T. and Sasaki I., Stability of discrete ground state. Hokkaido Math. J., 34(3) (2005), 689-717.

[9] Nelson E., Interaction of nonrelativistic particles with a quantized scalar field. J. Mathematical Phys., 5 (1964), 1190-1197.

[10] Reed M. and Simon B., Methods of modern mathematical physics. II. Fourier analysis, self-adjointness, Academic Press, New York, 1975.

[11] Reed M. and Simon B., Methods of modern mathematical physics. I, Functional analysis, second edition, Academic Press, New York,1980.

Department of Mathematics

Hokkaido University

Sapporo 060-0810, Japan

E-mail: teranishi@math.sci.hokudai.ac.jp 